\title{
Blind anti-collision methods for RFID system: a comparative analysis
}

\author{
Chaofu Jing, Zhongqiang Luo, Yan Chen, and Xingzhong Xiong
}

\begin{abstract}
Radio Frequency Identification (RFID) is one of the critical technologies of the Internet of Things (IoT). With the rapid development of IoT and the extensive use of RFID in our life, the pace of RFID development should be increased. However, the tags in an RFID system are more and more utilized, all of them communicate in the same channel. The RFID reader receives mixed signals, and the reader cannot get the correct message the tags send directly. This phenomenon is often called a collision, which is the main obstacle to the development of the RFID system. Traditionally, the algorithm to solve the collision problem is called the anti-collision algorithm, the widely used anti-collision algorithm is based on Time Division Multiple Access (TDMA) like ALOHA-based and Binary search-based anti-collision algorithm. The principle of the TDMA-based anticollision algorithm is to narrow the response of tags to one in each query time. These anti-collision algorithms perform poorly when the number of tags is huge, thus, some researchers proposed the Blind Source Separation (BSS)-based anti-collision algorithm. The blind anti-collision algorithms perform better than the TDMA-based algorithms; it is meaningful to do some more research about this filed. This paper uses several BSS algorithms like FastICA, PowerICA, ICA_p, and SNR_MAX to separate the mixed signals in the RFID system and compare the performance of them. Simulation results and analysis demonstrate that the ICA_p algorithm has the best comprehensive performance among the mentioned algorithms. The FastICA algorithm is very unstable, and has a lower separation success rate, and the SNR_MAX algorithm has the worst performance among the algorithms applied in the RFID system. Some advice for future work will be put up in the end.
\end{abstract}

Index Terms-BSS, RFID, FastICA, ICA_P, PowerICA, SNRMax.

$\mathrm{R}$ adio Frequency Identification (RFID) plays an important role in future IoT applications. It consists of three parts, computer, reader, and tags [1,2]. All of the tags communicate with the reader through the same wireless channel [3], once more than one tags in the scope of the reader, the backscattering signals will be mixed randomly, thus, the reader cannot recognize the message the tags transmitted directly. To solve this problem, the reader must use specific methods to avoid the collision, i.e., anti-collision algorithm $[4,5,6]$.

This work was supported by National Natural Science Foundation of China (No. 61801319), the Opening Project of Key Laboratory of Higher Education of Sichuan Province for Enterprise Informationalization and Internet of Things (No. 2017WZJ01), Sichuan Outstanding Youth Fund Project (No. 2020JDJQ0061), the Education Agency Project of Sichuan Province (No.18ZB0419), and the Major Frontier Project of Science and Technology Plan of Sichuan Province (No. 2018JY0512).
RFID belongs to the sensor layer of IoT, various sensors connect to IoT through RFID [7,8]. As IoT is an important technology of future life, the RFID system is required to be faster and with high stability [9-12], which is a huge challenge. The anti-collision algorithm plays an important role of the RFID system, via robust anti-collision algorithms, the RFID system will perform better and match the IoT better.

The traditional anti-collision algorithms are ALOHA-based and Binary search-based anti-collision algorithms. Both of them are based on Time Division Multiple Access (TDMA). They are easy to apply, but the time cost of these algorithms is high and the tags in such a system may not be identified in some cases [13-15]. The rule of the TDMA-based anti-collision algorithms is narrowing the tag's response to one in each query time. The RFID system uses these anti-collision algorithms will query and response several times, in some low Signal Noise Ratio (SNR) channel, the tags may be lost because of the silent command of the reader [16]. The maximum throughput of the RFID system using the dynamic frame slotted Aloha (DFSA, one of the TDMA-based anti-collision algorithm) is only $42.6 \%$ [17], and the maximum throughput of the RFID system using the Binary-tree searching of regressive index anti-collision algorithm is lower than $50 \%$ [18]. To get better performance, some researchers proposed the anti-collision algorithms based on the FastICA algorithm $[19,20]$. The RFID systems use these algorithms received a better result. the throughput of these systems is up to $69 \%$ of the highest [21-26], but the performance is not equal to expectation, the system uses FastICA algorithm performance bad in a low SNR channel, and the tag may not be identified even in a high SNR channel [27].

This paper aiming to find the fast and stable blind algorithms which can separate the RFID system mixed-signal well. This paper cites some BSS methods like PowerICA, ICA_p, and SNR MAX to the RFID system, and simulate in the computer via MATLAB. The performance of the algorithms can be represented by the Similarity between Source and Results (SSR) [22]. When the SSR is bigger than 0.92, we believe that separation is a success. The Success Rate (SR) can represent the performance in another form. We hypothesize the SNR of the channel, and the length of the tags can influence the performance of the anti-collision algorithms. So, we do the simulation in these two aspects.

Chaofu Jing, Zhongqiang Luo, Yan Chen and Xingzhong Xiong are with Artificial Intelligence Key Laboratory of Sichuan Province, Sichuan University of Science and Engineering, Yibin 644000, China(757949023@qq.com).

Zhongqiang Luo is also with Key Laboratory of Higher Education of Sichuan Province for Enterprise Informationalization and Internet of Things, Sichuan University of Science and Engineering, Yibin 644000, China (Corresponding author: zhongqiangluo@gmail.com) 
The rest of the paper is structured as follows. In section 2, the model of the RFID system and collision in the RFID system will be constructed. The collision problem will be elaborated. In section 3, we will introduce the theory of FastICA, PowerICA, ICA_p, and SNR_MAX. In section 4, the simulation of these mentioned algorithms will be implemented. In section 5, the result of the simulation will be brought up and analyzed, and some advice for future anti-collision work will be suggested.

\section{SYSTEM MODEL AND PROBLEM FORMULATION}

The RFID system consists of three parts: the computer the reader and the tags. The reader sends the order and the energy through the Radio Frequency (RF) channel to the tags. The tags send back the data to the reader through the RF channel. Then the reader sends the data to the computer connected to it. The computer handles these signals from the reader. Figure 1 shows the traditional model of the RFID system. Generally, the RFID systems have no more than 8 antennas in one reader, and with hundreds or thousands of tags [4]. The reader is expected to identify hundreds of tags in a short time in real life, and this made the model becoming an under-determined model. However, the algorithms we used are both only matches a determined or over-determined model, so we need to divide the tags into several groups then separate the mixed signals of every group.

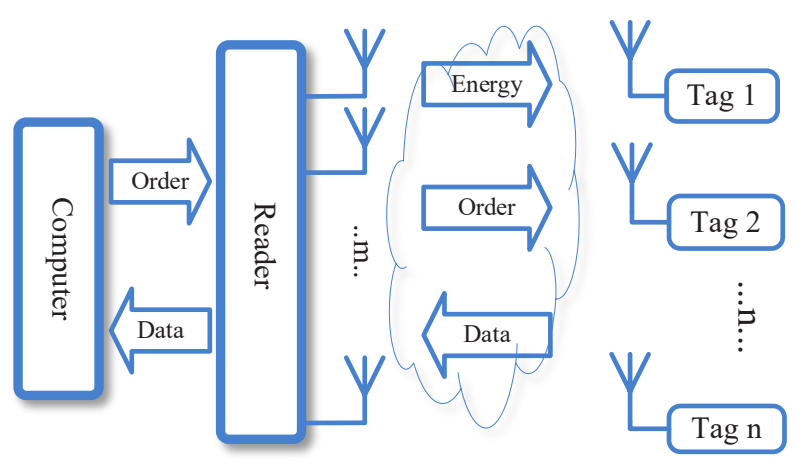

Fig. 1. RFID model

Assume that there are $m$ reader antennas, the received signals are $\mathbf{X}=\left[\mathbf{x}_{1}, \mathbf{x}_{2}, \ldots, \mathbf{x}_{\mathrm{m}}\right]^{\mathrm{T}}, \mathbf{x}_{1}, \mathbf{x}_{2}, \ldots, \mathbf{x}_{\mathrm{m}}$ is the received signal vector of each reader antenna. Suppose that each group has $n$ tags, the unknown signals of the $\mathrm{n}$ tags are $\mathbf{S}=\left[\mathbf{s}_{1}, \mathbf{s}_{2}, \ldots, \mathbf{s}_{\mathrm{n}}\right]^{\mathrm{T}}$, where $\mathbf{s}_{1}, \mathbf{s}_{2}, \ldots, \mathbf{s}_{\mathrm{n}}$ is the source signal vector of each tag. After the source signal $\mathbf{S}$ transmits through the RF channel, the signals may be randomly mixed, and the received signals $\mathbf{X}$ are far different from $\mathbf{S}$, we presume the mixing matrix is $\mathbf{M}^{m \times n}$. Then the relation between $\mathbf{X}$ and $\mathbf{S}$ is:

$$
\mathbf{X}=\mathbf{M S}+\mathbf{n}
$$

The received signals $\mathbf{X}$ cannot be processed by the traditional reader, in other words, the collision has happened. The $\mathbf{n}$ is the noise matrix, it is white Gaussian noise usually. We can see the model of collision from Figure 2.

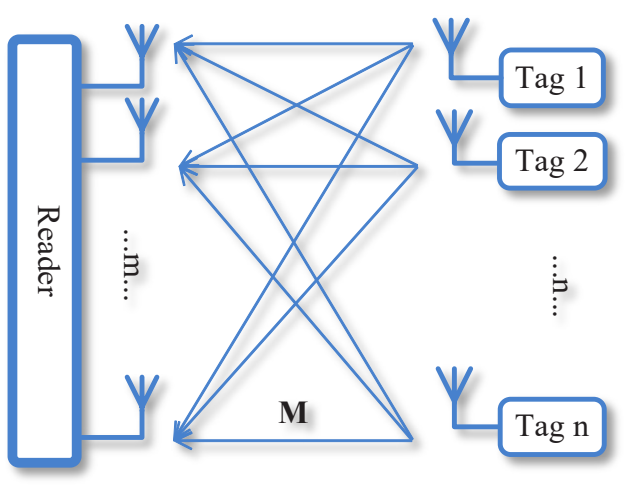

Fig. 2. Collision model

We can see from Figure 2, each antenna of the reader will receive the weighting sum of all tag's signal, as the RF channel is uncertain, we cannot know the weight of each tag's signal respectively, in other words, the $\mathbf{M}$ in Figure 2 is unknown. So, the signals cannot be identified by the readers without an anticollision algorithm. The traditional way to solve this problem is to avoid this mixing by identifying the tags one by one. It will increase the identification time and reduce the efficiency of the RFID system. Figure 3 shows the traditional anti-collision method.

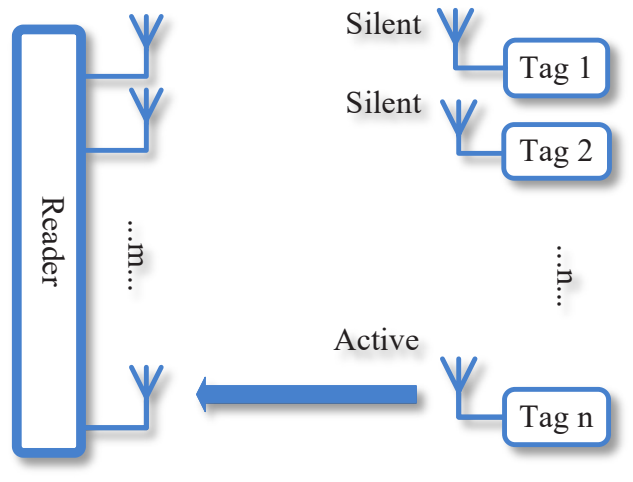

Fig. 3. Traditional anti-collision method

BSS is a data-driven signal processing method which posed in the 1980s [28,29]. It involves extracting and recovering the underlying source signals from multivariable statistical data. The source signal is unknown and either the mixing process is unknown in advance. There is only a small amount of prior knowledge such as statistical independence of source signal, the distribution of the source signal is at most one Gaussian distribution.

The source signal is the wave the tag backscattered we don't know before, and the mixed process in the wireless channel is unknown too. The source signal generated by the interior circuit of each tag, thus, it is statistically independent. The distribution of the source signal is non-Gaussian distribution. So, the mixed signal of the RFID system can be separated by the BSS methods. 
Blind anti-collision methods for RFID system:

a comparative analysis

The BSS algorithms can calculate a de-mixing matrix $\mathbf{W}$ by the received signal $\mathbf{X}$, which can make the equation:

$$
\mathbf{W M}=\mathbf{I}
$$

I in equation (2) is an identity matrix. It is equal to the equation:

$$
\mathbf{W} \approx \mathbf{M}^{-1}
$$

Denote the signal we separated as $\mathbf{Y}$, then we get the equation:

$$
\mathbf{Y}=\mathbf{W X}=\mathbf{W M S} \approx \mathbf{I S}=\mathbf{S}
$$

Use the BSS algorithms to separate the mixed signal of RFID system. We can identify several tags in each time, it can save a lot of time and improve the efficiency of the RFID system significantly. We can see the new anti-collision method in Figure 4.

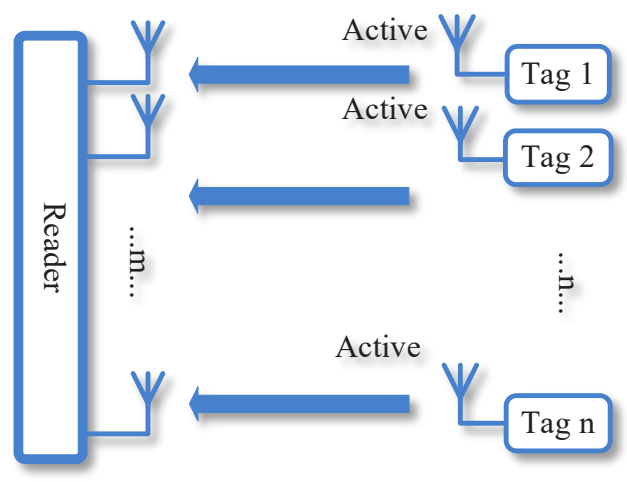

Fig. 4. BSS anti-collision method

\section{BSS ALGORITHMS}

BSS algorithms is a huge Data-driven signal processing algorithm family, include several algorithms such as Principle Component Analysis (PCA), Independent Component Analysis (ICA), Non-negative Matrix Factorization (NMF), Sparse Component Analysis (SCA) and so on [28]. The ICA algorithm is the presentation of BSS algorithms, in the next work, the traditional FastICA algorithm and the improved ICA_p and ICA_p algorithm will be introduced. The SNR-MAX algorithm is also included for its simplification to make a comparation.

\section{A. ICA method}

ICA is mainly used to solve the problem of BSS [29]. In cases where the source signal and mixing matrix are unknown. To make sure the ICA algorithm can separate the source signals from the mixed signals well, merely assuming that the source signals are statistically independent [27]. The ICA algorithm has an assumption that: (1) source signals are real random variables with zero means and are statistically independent of each other; (2) the number of source signals $n$ should be less than or equal to the number of observed signals $m(n \leq m)$. The mixing matrix $\mathbf{M}$ is a $\mathrm{m} \times \mathrm{n}$ unknown matrix with full rank; (3) At most one source signal is allowed to satisfy the Gaussian distribution. The schematic block diagram of the ICA is depicted in Figure 5.

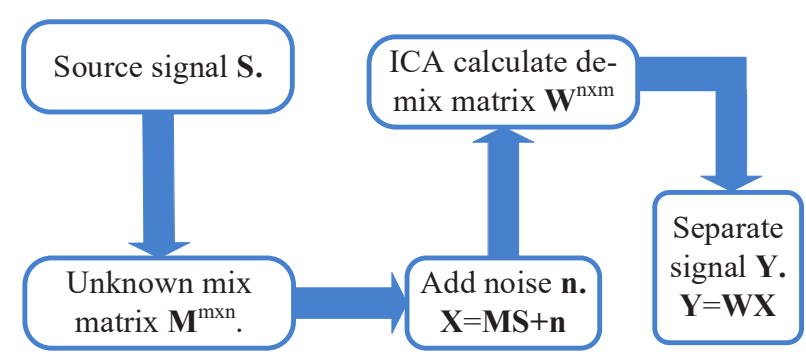

Fig. 5. Block diagram of ICA

The ICA method minimizes the statistical dependence between each component of the signal, highlights the essential structure of the source signal. One of the most popular ICA algorithms is the FastICA fixed-point algorithm [28], and the FastICA algorithm has a requirement that the data should be centered and pre-whitened. We can subtract the mean of the received signal to center the data.

$$
\mathbf{X}=\mathbf{X}-\mathrm{E}(\mathbf{X})
$$

$\mathrm{E}($.$) is the operator to get the expectation. The whitening$ process [28] can be finished by the following steps: First, we calculate the eigenvalues: $\mathbf{E}=\left(e_{1}, e_{2}, \ldots, e_{m}\right)$ and eigenvectors $\mathbf{D}=\left(d_{1}, d_{2}, \ldots, d_{m}\right)$ of the received data, Then the whitening matrix can be calculated by:

Then the whitened data:

$$
\mathbf{T}=\mathbf{D}^{-1 / 2} \mathbf{E}^{\mathrm{T}}
$$

$$
\mathbf{Y}_{1}=\mathbf{T X}
$$

Then the data can be separated successfully by the FastICA algorithm. The FastICA algorithm finds the de-mixing matrix $\mathrm{W}$ by the iteration objective function:

$$
J(\mathbf{y}) \approx \sum_{i=1}^{p} k_{i}\left\{\mathrm{E}\left[G_{i}(\mathbf{y})\right]-\mathrm{E}\left[G_{i}(\mathbf{v})\right]\right\}^{2}
$$

Where $k_{i}$ is a positive constant and $v$ is a random variable of the Gaussian with zero mean and unit variance. $G_{i}(\bullet)$ is a nonquadratic function, the select of $\mathrm{G}$ is different of the Gaussian distribution [28]. As the RFID signals are sub-Gaussian so the $\mathrm{G}$ can be chosen as follows:

$$
\begin{aligned}
& G(\mathbf{u})=\frac{1}{a_{1}} \log \cosh \left(a_{1} \mathbf{u}\right), G^{\prime}(\mathbf{u})=\tanh \left(a_{1} \mathbf{u}\right) \\
& 1 \leq a_{1} \leq 2
\end{aligned}
$$

Then maximizes the Lagrangian:

$$
L(\mathbf{w}, \lambda)=\left|\mathrm{E}\left[G\left(\mathbf{w}^{\mathrm{T}} R\right)\right]\right|-\frac{\lambda}{2}\left(\mathbf{w}^{\mathrm{T}} \mathbf{w}-1\right)
$$

\section{B. PowerICA method}

The ICA algorithm has some drawbacks, such as: The fixedpoint ICA algorithm can only do a serial computation. Some unnecessary assumptions in the iteration of the FastICA algorithm are exist. The classical derivation of the FastICA algorithm has difficulty in separating large sets of real data quickly and accurately. To solve the problems mentioned before, the PowerICA and the ICA_p algorithm have been proposed respectively. 
Shahab Basiri [27] provided a novel power iteration algorithm for FastICA which is remarkably more stable when the sample size is not orders of magnitudes larger than the dimension. And the PowerICA algorithm can be run on parallel computing nodes. Basiri cut the oversimplified assumptions of the Lagrangian and the Jacobian matrix in the FastICA algorithm and put up with a new power iteration method for FastICA. The new PowerICA algorithm can converge in the case of the $n=m$, and drastically reduce the computational time by a run on parallel computing nodes. They change the Lagrangian to:

$$
\mathrm{L}(\mathbf{w}, \lambda)=\left|\mathrm{E}\left[G\left(\mathbf{w}^{\mathrm{T}} \mathrm{R}\right)\right]\right|-\frac{\lambda(\mathbf{w})}{2}\left(\mathbf{w}^{\mathrm{T}} \mathbf{w}-1\right)
$$

Thus, the algorithm solving:

$$
\mathrm{F}(\mathbf{w})=m(\mathbf{w})-\lambda(\mathbf{w}) \mathbf{w}=0
$$

Iterates:

$$
\mathbf{w} \leftarrow \frac{m(\mathbf{w})-\beta(\mathbf{w}) \mathbf{w}}{\|m(\mathbf{w})-\beta(\mathbf{w}) \mathbf{w}\|}
$$

Until convergence. The $\beta(\mathbf{w})$ in (13) is a scalar multiplier defined as $\beta(\mathbf{w})=\mathrm{E}\left[g^{\prime}\left(\mathbf{w}^{\mathrm{T}} \mathbf{x}\right)\right] \in \mathrm{R}$.

\section{ICA_p method}

Pierre Ablin [30] introduced a Preconditioned ICA for the Real Data algorithm, which is a relative L-BFGS algorithm preconditioned with sparse Hessian approximations. They found that the Hessian approximations have a low cost per iteration but not accurate enough on real data. To solve this problem, the use of an optimization algorithm which 'learns' curvature from the past iterations of the solver, and accelerates it by preconditioning with Hessian approximations. They put the Hessian approximations as follows:

$$
\left\{\begin{array}{c}
\hat{h}_{i j l}=\hat{\mathrm{E}}\left[\varphi_{i}^{\prime}\left(\mathbf{y}_{i}\right) \mathbf{y}_{j} \mathbf{y}_{l}\right], \text { for } 1 \leq i, j, l \leq \mathrm{N} \\
\hat{h}_{i j}=\hat{\mathrm{E}}\left[\varphi_{i}^{\prime}\left(\mathbf{y}_{i}\right) \mathbf{y}_{j}^{2}\right], \text { for } 1 \leq i, j \leq \mathrm{N} \\
\hat{h}_{i}=\hat{\mathrm{E}}\left[\varphi_{i}^{\prime}\left(\mathbf{y}_{i}\right)\right], \text { for } 1 \leq i \leq \mathrm{N} \\
\delta_{i}^{2}=\hat{\mathrm{E}}\left[\mathbf{y}_{i}^{2}\right], \text { forl } \leq i \leq \mathrm{N}
\end{array}\right.
$$

They denote the approximation by $\tilde{H}^{2}$ in the first step:

$$
\tilde{H}_{i j k l}^{2}=\delta_{i l} \delta_{j k}+\delta_{i k} \delta_{j l} \hat{h}_{i j}
$$

Then denoted $\tilde{H}^{1}$, goes one step further and replaces $\hat{h}_{i j}$ by

$$
\begin{gathered}
\hat{h}_{i} \hat{\sigma}_{j}^{2} \text { for } i \neq j: \\
\left\{\begin{array}{c}
\tilde{H}_{i j k l}^{1}=\delta_{i l} \delta_{j k}+\delta_{i k} \delta_{j l} \hat{h}_{i} \hat{\sigma}_{j}^{2} \\
\tilde{H}_{i i i i}^{1}=1+\hat{h}_{i i}
\end{array}\right.
\end{gathered}
$$

They designed an algorithm to precondition the data based on the Hessian approximation mentioned before.

\section{SNR-Max method}

ZHANG Xiao-bing [24] proposed a low computational complexity instantaneous liner mixture blind separation algorithm. They take care of the complexity of the traditional algorithms, eye on the character that SNR is maximal when statistically independent source signals are entirely separated. They expressed the source signals and noise to the generalized eigenvalue problem, and get de-mixing matrix without iteration. They use the difference between the source signal and the estimated signal: $\mathbf{e}=\mathbf{s} \mathbf{- y}$ as a noise signal. Then get the SNR function:

$$
S N R=10 \log \frac{s \bullet s^{\mathrm{T}}}{e \bullet e^{\mathrm{T}}}=10 \log \frac{s \bullet s^{\mathrm{T}}}{(s-y) \bullet(s-y)^{\mathrm{T}}}
$$

But the source is unknown, so the use the moving average $\tilde{y}$ of the signal to replace the source signal $s$, so the SNR changes to:

$$
S N R=10 \log \frac{s \bullet s^{\mathrm{T}}}{e \bullet e^{\mathrm{T}}}=10 \log \frac{\tilde{y} \bullet \tilde{y}^{\mathrm{T}}}{(\tilde{y}-y) \bullet(\tilde{y}-y)^{\mathrm{T}}}
$$

After some simplification, the final objective function is:

$$
F(y)=S N R=10 \log \frac{y \bullet y^{\mathrm{T}}}{(\tilde{y}-y) \bullet(\tilde{y}-y)^{\mathrm{T}}}
$$

They use a gradient descent method to find the maximum value of the object function, the get the suitable de-mixing matrix w.

\section{SimUlation ANALYSiS AND DisCUSSION}

We use computer software MATLAB to do the simulation. We set a RFID system with $m$ reader antennas and $n$ tags in each group, the data length of tags is $\mathrm{k}$. Then the source signal noted as $\mathbf{S}^{n \times k}$, the received signal noted as $\mathbf{X}^{m \times k}$, the unknown mix process simulated by a randomly full rank matrix $\mathbf{M}^{m \times n}$, Then we multiply the $\mathbf{M}$ by $\mathbf{S}$, as the equation: $\mathbf{X}=\mathbf{M S}$. Then the algorithms mentioned before will be used to estimate the demix matrix $\mathbf{W}^{n \times m}$, we denote the signal we separate by the algorithms as $\mathbf{Y}$. then: $\mathbf{Y}=\mathbf{W X}$ The block diagram of the simulation process is shown in Figure 6.

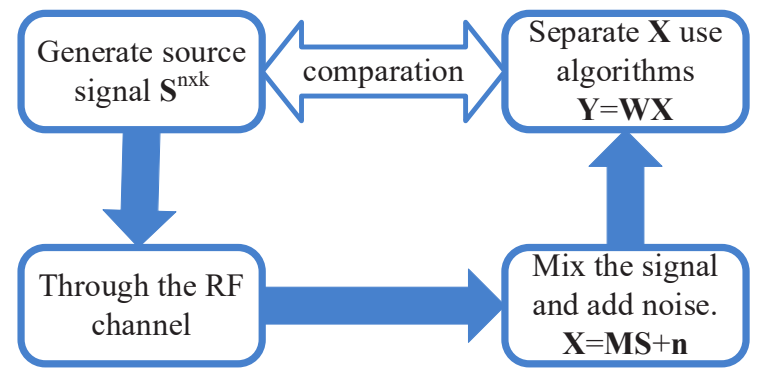

Fig. 6. Block diagram of the simulation process

This paper compares the result in SSR and SR change with the SNR. Secondly, compare the SSR and SR change with the data length of the tags. 
Blind anti-collision methods for RFID system:

a comparative analysis

Aiming to get a better comparison, we set a RFID system with 5 reader antennas and 5 tags need to be separated each time. When compare the SSR and SR change with the SNR, we set the data length of the tags to 1000 , the SNR change from 0 to 30, simulation results are shown in Figure 7 and Figure 8. When compare the SSR and SR change with the data length of the tags, we set SNR to 25, the data length of tags change from 500 to 5000 with a step of 500, the results are shown in Figure 9 and Figure 10. All of the data are an average of 3000 times.

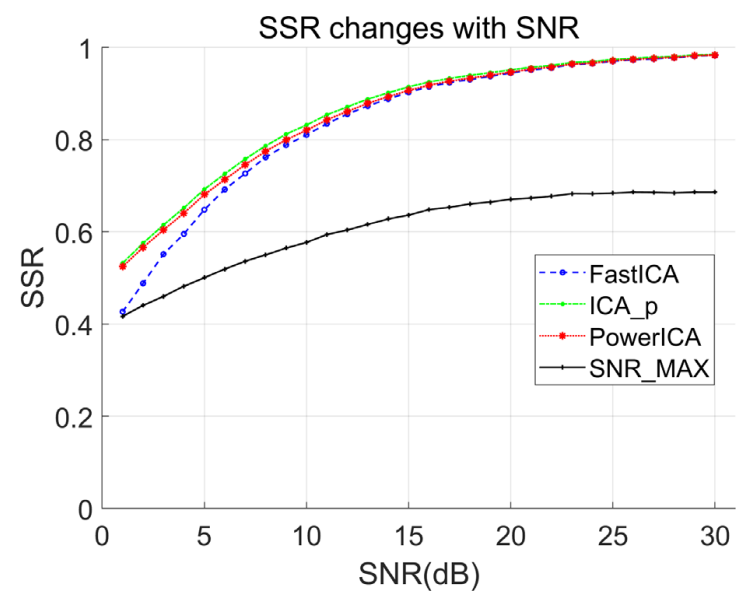

Fig. 7. SSR changes with SNR

In Figure 7, firstly, the trend of SSR changes with SNR is correct. Generally, when the channel's SNR is up to 20, the SSR is more than 0.92 , it is a very good performance. When the channel's SNR is less than 20, the performance of the ICA_p algorithm is the best, and the PowerICA algorithm is as good as the ICA_p algorithm but just a little bit worth than the later, the FastICA algorithm has a low SSR, especially in the low SNR channel. Actually, the FastICA algorithm is not stable, the iteration of the FastICA algorithm may not convergence, we deleted the data not convergence then draw Figure 7 and Figure 8. The performance of the SNR_MAX is very poor no matter the condition of channel is good or bad, we believe the low computational complexity of the SNR_MAX algorithm may explain this result. In conclusion, the ICA_p has the best comprehensive performance. The PowerICA algorithm has almost the same performance with the ICA p algorithm. FastICA performnot so good in a channel with low SNR, and it is not stable. The SNR_MAX algorithm can not meet the requirement of the RFID system.

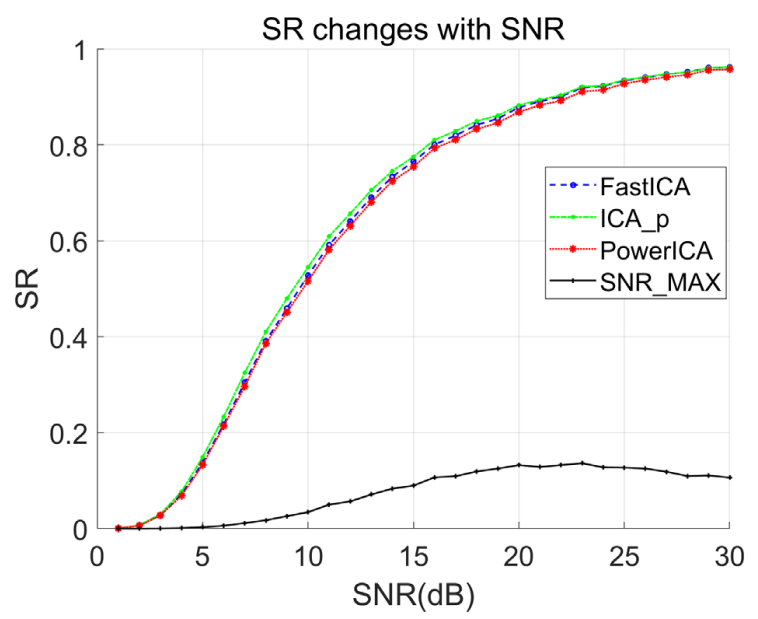

Fig. 8. SR changes with SNR

The paper also compared the SR changes with the channel's SNR, the result is shown in Figure 8. Usually, if the SSR is bigger than 0.92 , we claim the separate is a success. In the curve, we found the FastICA, ICA p, PowerICA algorithm have the related SR trend, but the ICA_p algorithm performance a little better. The SNR_MAX algorithm is hard to satisfy the system.

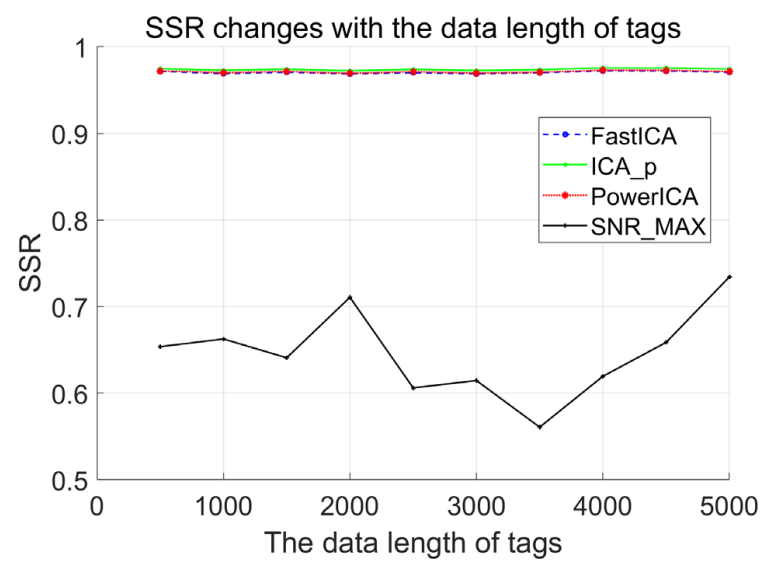

Fig. 9. SSR changes with the data length of tags

From Figure 7 and 8, we can find that the curve becomes smooth when the SNR of the channel is more than 25 and the performance of the algorithms are both very good, which means the SNR in this level make no much difference of the SSR. So, we set the SNR to 25 , then compare the algorithms' performance in different data length of tags. Figure 9 shows the performance, it tells the trues that all of the algorithms do well when the length of the tags change from 500 to 5000 . Because of the relation between the SSR and the SR, we believe that the success rate has the same trend as the correlation. Figure 10 verify the guess. 


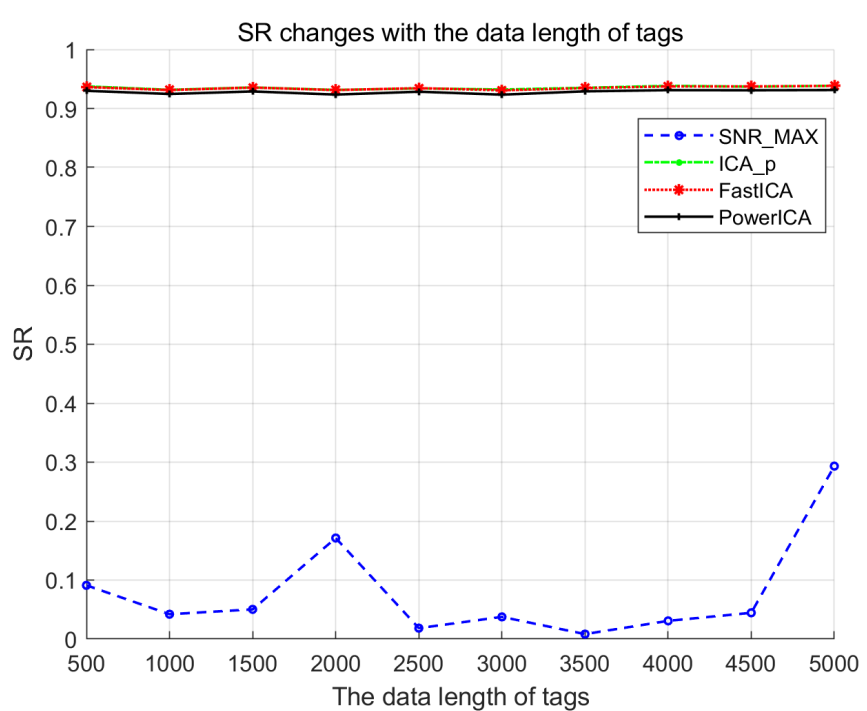

Fig. 10. SR changes with the data length of tags

As we said before, the FastICA algorithm is unstable, Figure 11 shows the FastICA algorithm default convergence time changes with the SNR, even when the SNR more than 20, the FastICA emerge the case of not convergence, but the PowerICA and the ICA_p will not occur such thing. The reason why this situation arises is that the algorithm oversimplified the Lagrangian and the Jacobian Matrix [26].

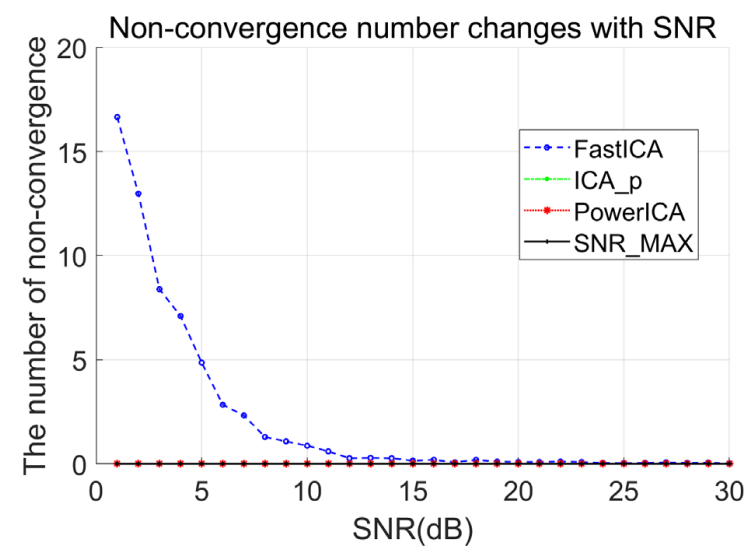

Fig. 11. The non-convergence time changes with SNR

By the way, we compared the time cost of the algorithms changes with the length of the tags, we can see it in Figure 12. The SNR_Max uses the least of time, and the Power needs the most of the time, the others between them. But the PowerICA can run in a parallel model, so it can perform better in parallel systems.

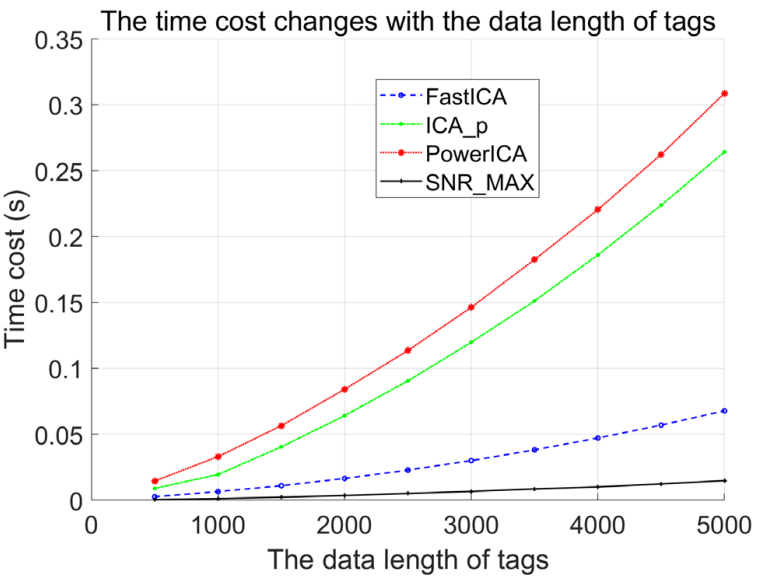

Fig. 12. the time cost changes with the length of tags

To show the BSS algorithm's separate performance visually, we put the source signal, the mixed-signal, and the algorithms separate the signal in Figure 13 to 18. Considering the comfort of view, we decline the number of tags to 3 , and only draw the first 20 data of the signal.

\section{Source signals}
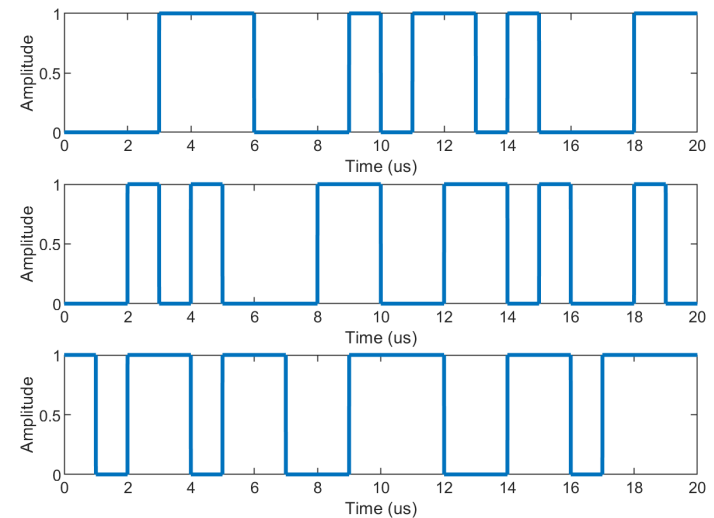

Fig. 13. The source signals

Mixed signals
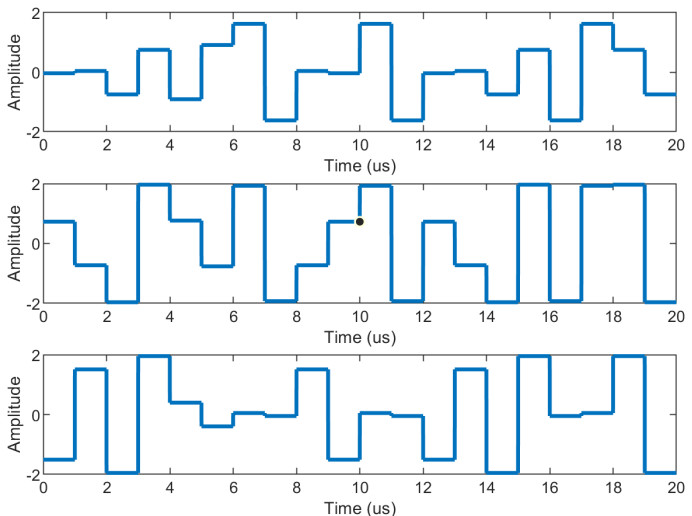

Fig. 14. The mixed signals 
Blind anti-collision methods for RFID system:

a comparative analysis

ICA_p separate signals
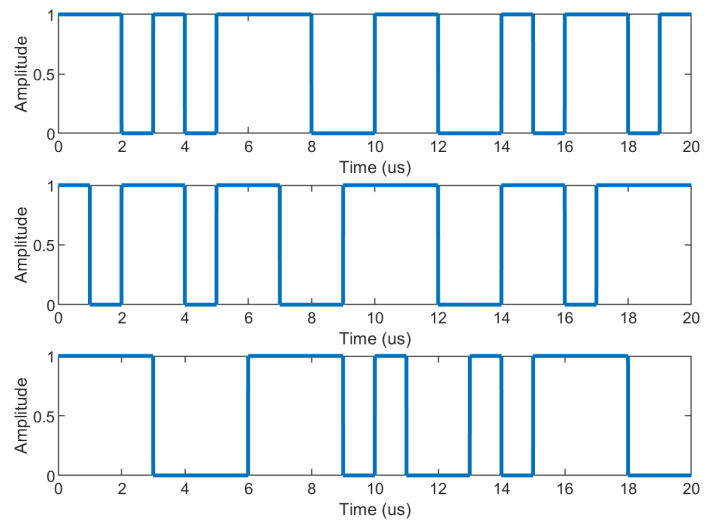

Fig. 15. The ICA_p separate signals

FastICA separate signals
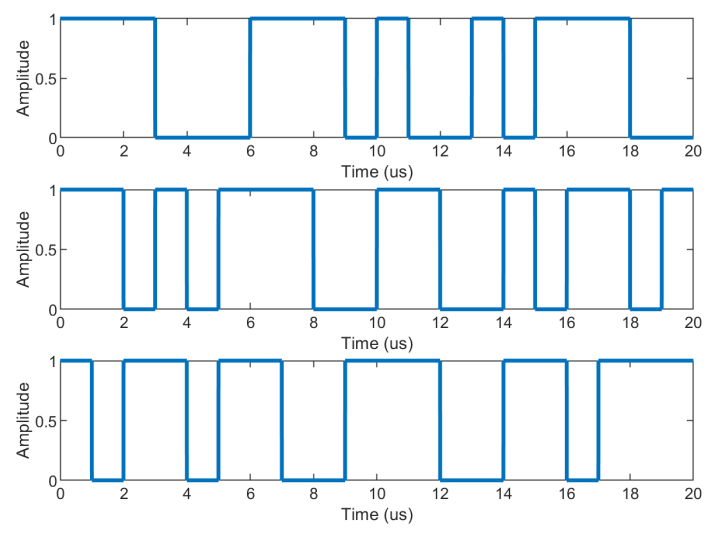

Fig. 16. The FastICA separate signals

PowerlCA separate signals
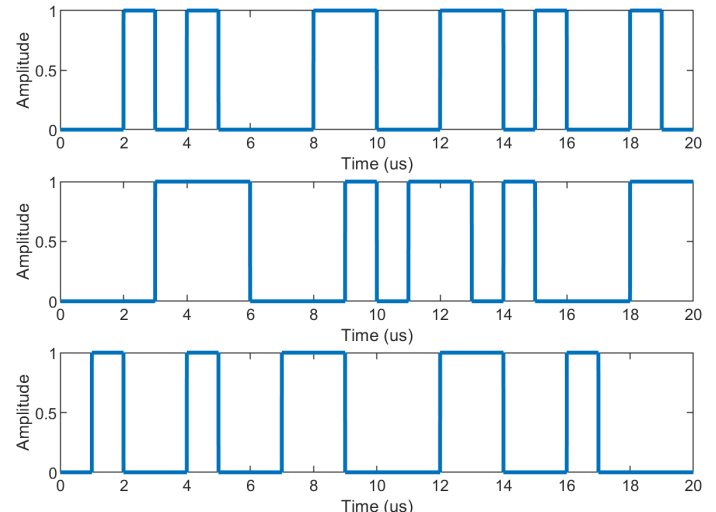

Fig. 17. The PowerICA separate signals
SNR_MAX separate signals
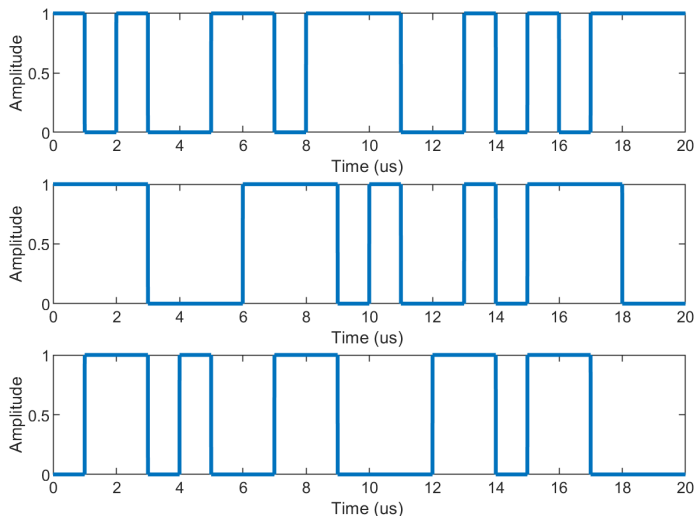

Fig. 18. The SNR-Max separate signal

We can see from Figure 12 to Firure 18 that the BSS methods change the order and the polarity of the signal, and most of the signal be separated successfully. The first signal of source (Figure 13) corresponds to the third signal of ICA p separate signals (Figure 15) with the opposite polarity, corresponds to the first signal of the FastICA separate signals (Figure 16) with the opposite polarity, corresponds to the second signals of PowerICA separate signals (Figure 17), however we cannot find the corresponding signal from the SNR MAX separate signals. As the use of FM0 coding in RFID system, the polarity and the order change of the signals doing nothing for us to recognize the information the tags send.

We make a table to show the performance of the algorithms we used. Shown as Table 1.

TABLE I

ALGORITHMS PERFORMANCE

\begin{tabular}{llll}
\hline \hline Algorithm & Accuracy & Stability & instantaneity \\
\hline FastICA & Medium & Low & High \\
PowerICA & High & High & Medium \\
ICA_p & High & High & Medium \\
SNR_MAX & Low & medium & High \\
\hline \hline
\end{tabular}

There are some useful simulation data to prove our conclusion. The data in Table 2 got in the channel which has a 25 SNR and the tags' length is 1000 . We do the simulation 1000 times, then get the average data. As we can see the ICA_p algorithm has the highest correlation in this situation. From the low correlation and the mean success rate we can say that the ICA_p and the PowerICA algorithm are stable. From the time for one separation, we know the Fast ICA algorithm is the most real-time and with excellent performance. 
TABLE II

SOME USEFUL DATA

\begin{tabular}{lllll}
\hline \hline Algorithm & $\begin{array}{l}\text { Highest } \\
\text { correlation }\end{array}$ & $\begin{array}{l}\text { Low } \\
\text { correlation }\end{array}$ & $\begin{array}{l}\text { Mean } \\
\text { successes } \\
\text { rate }\end{array}$ & $\begin{array}{l}\text { Time for } \\
\text { one } \\
\text { separate }\end{array}$ \\
\hline FastICA & 0.9952 & 0.8222 & 0.9680 & $0.0053 \mathrm{~s}$ \\
PowerICA & 0.9970 & 0.8662 & 0.9680 & $0.0176 \mathrm{~s}$ \\
ICA_p & 0.9999 & 0.8439 & 0.9680 & $0.0126 \mathrm{~s}$ \\
SNR-Max & 0.8055 & 0.5302 & 0.3440 & $0.0006 \mathrm{~s}$ \\
\hline \hline
\end{tabular}

RFID is located at the sensor layer of IoT, it has an important role of IoT. As IoT develops rapidly, the RFID system is required to be more effective and real-time in practical applications. The simulation of the paper shows that the FastICA algorithms perform well in real-time but has poor stability performance. The PowerICA and the ICA_p algorithms perform well in stability and accuracy, but need a little more time. And the SNR-Max algorithm is not suitable for the RFID system. In conclusion, we can design a system that uses ICA_p algorithm or PowerICA algorithm when the channel's SNR is low and use the FastICA algorithm to reduce the time when the channel's SNR is very high. The ICA_p algorithm has the best general performance.

This work confirms the feasibility of using BSS methods as anti-collision algorithms in RFID systems, verifies the good performance of BSS methods like ICA_p and PowerICA. However, our current work only takes care of over-determined or determined receiving model. The meaningful and practical underdetermined receiving model needs more attention from researchers. In future work, the underdetermined receiving model will be engaged for anti-collision in RFID application.

\section{REFERENCES}

[1] Want, and R. "An Introduction to RFID Technology." IEEE Pervasive Computing, vol. 5, no.1, pp. 25-33. Jan. 2006. Dor: 10.1109/MPRV.2006.2.

[2] Azambuja, Marcelo \& Marcon, César \& Hessel, F.. (2008). Survey of Standardized ISO 18000-6 RFID Anti-collision Protocols. 468 - 473. DOI: 10.1109/SENSORCOMM.2008.124.

[3] Quan CH., Hong WK., Kim HC. (2006) "Performance Analysis of Tag Anti-collision Algorithms for RFID Systems". In: Zhou X. et al. (eds) Emerging Directions in Embedded and Ubiquitous Computing. EUC 2006. Lecture Notes in Computer Science, vol 4097. Springer, Berlin, Heidelberg. Dor: 10.1007/11807964_39

[4] Jia, Xiaolin, Q. Feng, and L. Yu. "Stability Analysis of an Efficient Anti-Collision Protocol for RFID Tag Identification." IEEE Transactions on Communications vol. 60, no. 8, pp.2285-2294.

[5] Dacuña, Javier \& Melià-Seguí, Joan \& Pous, Rafael. (2012). "Multitag spatial multiplexing in UHF RFID systems". IEICE Electronics Express. 2012 vol.9, no. 21,pp. 1701-1706. DoI: 10.1587/elex.9.1701.

[6] Mota, Rafael \& Batista, Daniel. (2013). A RFID QoS mechanism for IoT tracking applications. 2013 International Symposium on Wireless and Pervasive Computing, ISWPC 2013. 1-4. DoI: 10.1109/ISWPC.2013.6707429.
[7] Zhang, Dacheng \& Liu, Yu \& Han, Kun \& Liu, Aiyong \& Liu, Lijing. (2012). The Application of RFID-Based on IOT in Logistics Management. Dor: 10.1007/978-3-642-29455-6_97.

[8] Alsinglawi, Belal \& Nguyen, Quang \& Gunawardana, Upul \& Simoff, Simeon \& Maeder, Anthony \& Elkhodr, Mahmoud \& Alshehri, Mohammad. (2019). Passive RFID Localization in the Internet of Things. DoI: 10.1007/978-3-319-99966-1_7.

[9] Kumar, Sachin, P. Tiwari, and M. Zymbler. "Internet of Things is a revolutionary approach for future technology enhancement: a review." Journal of Big Data 6.1(2019):1-21. DoI: 10.1186/s40537-019-0268-2.

[10] Chen S, Xu H, Liu D, et al. "A Vision of IoT: Applications, Challenges, and Opportunities with China Perspective." IEEE Internet of Things Journal, vol. 1, no. 4, pp. 349-359. 2014. DoI: 10.1109 /jiot.2014.2337336.

[11] Khan, Sarfaraz . "Health care monitoring system in Internet of Things (IoT) by using RFID." IEEE's 6th International Conference on Industrial Technology and Management (ICITM 2017) IEEE, 2017. DoI: 10.1109/ICITM.2017.7917920.

[12] Li Xue-qiao, JIA Xiao-ai, ZHAO Lei, XU Ben-fu. "Anti-collision Algorithm Based on Dynamic Searching of Regressive Index." Communications technology. Vol. 42, no. 06, pp. 118-120. 2009. DoI: $10.3969 /$ j.issn.1002-0802.2009.06.041.

[13] Xu Haifeng, Jiang Hui, "Research and Simulating on ALOHA Anticollision Algorithm with Higher Efficiency in Real-time for RFID System." Computer and Digital Engineering, vol. 39, no. 05, pp.1922+26. 2011

[14] Z. Xiao-Hong, Liu-Yang Z. "Research on Passive RFID System Adaptive Frame Slot Anti-collision Algorithm." Acta Electronica Sinica. Vol. 044 no.009. pp. 2211-2218. 2016. Dor: $10.3969 /$ j.issn.0372-2112.2016.09.028.

[15] HAN Xianming NAN Jingchang, "Anti-Collision Algorithm for RFID Based on Regressive Index Binary-Tree Searching." Microelectronics, vol. 43, no. 05, pp. 708-712. 2013.

[16] M. Vedhekar, Sairam A S, Kumari A. "Binary countdown anticollision protocol for RFID tag collision problem." 2016 International Conference on Accessibility to Digital World (ICADW). IEEE, 2016. DoI: 10.1109/ICADW.2016.7942522.

[17] Jiang Xia, Bai Tiecheng Zheng Hongjiang, "Research on Binary Search Anti-collision Algorithm for RFID System." Journal of Tarim University, vol. 25, no. 04, pp. 30-35, 2013.

[18] MU Yuchao ZHANG Xiaohong, "Adaptive tree grouping and blind separation anti-collision algorithm for radio frequency identification system." Journal of Computer Applications, vol. 35, no. 01, pp. 19-22, 2015. DoI: 10.1021/JM9005093.

[19] Nguyen C T, Hayashi K, Megumi Kaneko.... "Probabilistic Dynamic Framed Slotted ALOHA for RFID Tag Identification." Wireless Personal Communications, vol. 71, no. 4, pp. 2947-2963. 2013.

[20] LI Hua, JIA Zhi-ping, "UHF RFID anti-collision algorithm based on blind separation and dynamic bit-slot grouping." Journal on Communications, vol.33, no. 04, pp. 47-53. 2012.

[21] PENG Yong-hua HE Yi-gang, "Application of a New Type ICA Algorithm in RFID System." Computer Engineering, vol. 38, no. 19, pp. 25-29. 2012.

[22] L.I. Hua. W. Hong-jun and S. Zi-liang, "ICA-based UHF RFID Multitag Hybrid Data Blind Separation". in International conference on communication and electronics information. Haikou (CN). p. 52-56. 2013.

[23] Zhang Xiaohong Jin Yungang, "Research of Under-determined Blind Source Separation Anti-collision Algorithm Based on RFID Frame-slot." Journal of System Simulation, vol. 28, no. 05, pp. 11001108+1116. 2016.

[24] T. Chu, Zou S X, Ren W, et al. "DRICA design of multi-antenna RFID system based on ICA." International Workshop on Wireless Communication and Network (IWWCN2015). 2016. Dor: $10.1142 / 9789814733663 \_0044$ 
[25] P. Yong-Hua, Yi-Gang H E. "Application of a New Type ICA Algorithm in RFID System.” Computer Engineering. Vol. 38, no. 19, pp. 25-29 2012

[26] Basiri, S., E. Ollila and V. Koivunen, "Alternative Derivation of FastICA With Novel Power Iteration Algorithm." IEEE Signal Processing Letters, vol. 24, no. 9, pp. 1378-1382. 2017. DOI: $10.1109 / \mathrm{lsp} .2017 .2732342$.

[27] Luo, Z., C. Li and L. Zhu, "A Comprehensive Survey on Blind Source Separation for Wireless Adaptive Processing: Principles, Perspectives, Challenges and New Research Directions." IEEE Access, vol. 6, no. 6, pp. 66685-66708. 2018. Dor: 10.1109/ACCESS.2018.2879380.

[28] X. Yu, D. Hu, J. Xu, "Blind Source Separation: Theory and Applications", Singapore: John Wiley \& Sons, 2014.

[29] Ablin, Pierre, Jean-François Cardoso, and A. Gramfort . "Accelerating Likelihood Optimization for ICA on Real Signals." Latent Variable Analysis and Signal Separation. 2018. DoI: $10.1007 / 978-3-319-93764-9$ 15.

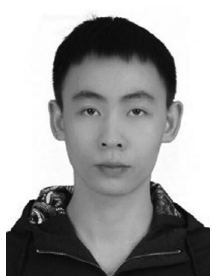

Chaofu Jing received the B. S. degree in Electronic and Information Engineering from Sichuan University of Science and Engineering (SUSE) Zigong, China, in 2018. Now he is a postgraduate student in Automation \& Information Engineering of SUSE. He will receive a M. S. degree in 2021. His research contain Blind Source Separation, Radio Frequency Identification, intelligent Signal Processing and Artificial Intelligence.

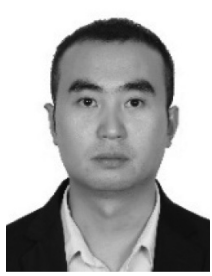

Zhongqiang Luo received the B.S. and M.S. degrees in communication engineering and pattern recognition and intelligent systems from Sichuan University of Science and Engineering, Zigong, China, in 2009 and 2012, respectively. He received the Ph.D. degree in communication and information systems from University of Electronic Science and Technology of China (UESTC), in 2016. Since 2017, he has been with the Sichuan University of Science and Engineering, where he is currently an associate professor. From December 2018-December 2019, he was a visiting scholar with Department of Computer Science and Electrical Engineering in University of Maryland Baltimore County (UMBC). His research interests include machine learning, blind source separation, signal processing for wireless communication system and intelligent signal processing.

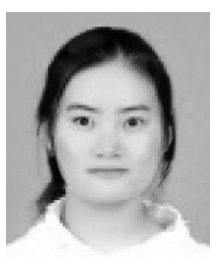

Chen Yan received the B.S degrees in communication engineering from Sichuan University of Science and Engineering, Zigong, China, in 2018. Since 2018, she has studying for a master's degree at Sichuan University of Science and Engineering, Yibin. Her research interests include blind source separation, signal processing for wireless communication system and intelligent signal processing.

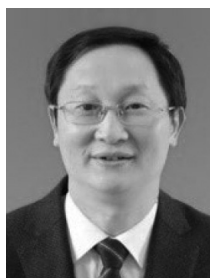

Xingzhong Xiong received the B.S. degrees in communication engineering from Sichuan University of Science and Engineering, Zigong, China, in 1996. He received the M.S and Ph.D. degrees in communication and information system from University of Electronic Science and Technology of China (UESTC), in 2006 and 2009, respectively. In 2012, he completed a research assignment from the Postdoctoral Station of Electronic Science and Technology at the UESTC. He is currently a professor at the School of Automation and Electronic Information, Sichuan University of Science and Engineering. His research interests include wireless and mobile communications technologies, intelligent signal processing, Internet of Things technologies, and very largescale integration (VLSI) designs. 